\title{
DISEÑO E IMPLEMENTACIÓN DE UN PROTOTIPO DE BRAZO ROBÓTICO EN 3D PARA LA MANIPULACIÓN DE CAJAS EN UNA MATRIZ DE ALMACENAMIENTO
}

Paola Andrea Rey Molina

Ingeniera de Seguridad

G4S Tecnology

paolitareymolina@hotmail.com

Bogotá, Colombia

\section{Karent Urrego}

Ingeniera de Seguridad

G4S Tecnology

kittyflower2009@hotmail.com

Bogotá, Colombia

Tipo: Artículo de investigación

Fecha de Recepción: Junio 19 de 2012

Fecha de Aceptación: Agosto 9 de 2012

\section{A 3-D ROBOTIC ARM FOR MANIPULATING BOXES WITHIN A STORAGE MATRIX - DESIGN AND IMPLE- MENTATION}

\begin{abstract}
This paper summarizes the results obtained from a robotic-arm prototype-development project. The robotic arm manipulates either substances or objects that are considered harmful for operators. The prototype is intended for mass production factories that require manipulation of potentially harmful objects or chemical substances.

Key words: manipulator, mechatronics, positioning, programming.

RESUMEN

A través de éste artículo se presentan los resultados obtenidos en el desarrollo del proyecto de un prototipo de brazo robótico que permite manipular sustancias u objetos que coloquen en riesgo la vida e integridad del operario, el proyecto está pensado y dirigido hacia empresas que manejen altos volúmenes de producción y que manipulen sustancias químicas u objetos que impliquen alto riesgo para el ser humano.
\end{abstract}

Palabras claves: manipulador, mecatrónica, posicionamiento, programación. 


\section{INTRODUCCIÓN}

El área de la robótica industrial es un campo amplio para el desarrollo de nuevas ideas, en el cual la variedad de aplicaciones que tienen los manipuladores es bastante diversa y que día a día busca optimizar y automatizar los procesos de manufacturación, haciendo más fácil y segura la vida del ser humano.

Teniendo en cuenta el amplio conocimiento con el que se cuenta en el área de la electrónica se desarrolló un prototipo de brazo robótico en 3D para la manipulación de cajas en una matriz.

El proyecto cuenta con dos áreas de desarrollo específicas como lo son el área mecánica y electrónica; el área mecánica se desarrolló a través de simulaciones virtuales con Solid Edge 20 y posteriormente se llevaron a físico, y el área electrónica se desarrolló a través del programa de PIC C, luego fue simulado en el entorno de proteus para finalmente llevar a cabo el hardware en el

\section{MATERIALES Y MÉTODOS}

Para la implementación del prototipo se construyó uno maqueta que simulara el entorno en el que el robot interactuaría, la maqueta está compuesta por tres módulos: Brazo robótico, interfaz de usuario y matriz de almacenamiento.

La interfaz de usuario es una caja de $32 \times 36$ $\mathrm{cm}$ en acrílico de $3 \mathrm{~mm}$ de espesor que tiene la función de dar estabilidad al brazo robótico e interactuar con el operario, ya que es en ella donde se encuentran la LCD, teclado, botón off/ on y los pulsadores de posicionamiento para el modo manual.

El robot tiene piezas en aluminio y en acrílico de acuerdo al requerimiento de torque, fuerza y estabilidad que exigió el sistema mecánico.

El desarrollo del sistema tiene a grandes rasgos tres grandes módulos, que se presentan en la figura 1.
- Diseño Mecánico

- Diseño Electrónico

- Integración de Hardware y Software
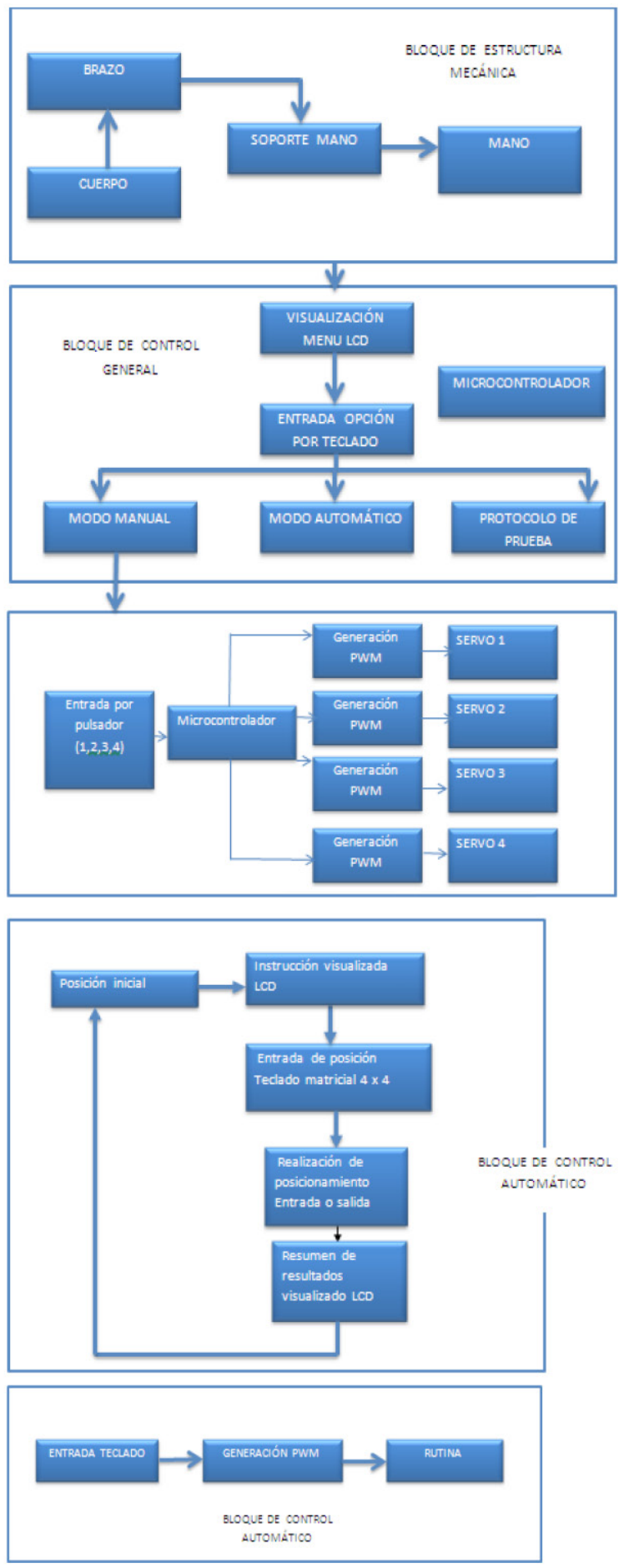

Figura 1. Diagrama de bloques del sistema. 
Los implementos utilizados en la elaboración del prototipo fueron seleccionados de acuerdo a las especificaciones, buscando dispositivos y elementos de excelentes características, óptimas condiciones y de bajo costo.

\subsection{Desarrollo mecánico}

El diseño del modelo mecánico del robot comienza por un simple manejo geométrico del esquema inicial del prototipo para crear el diseño final y total.

La cinemática directa permite analizar el posicionamiento de los elementos de un mecanismo articulado, teniendo en cuenta sus componentes fijas y los cambios que se generen por el movimiento de las articulaciones como se observa en la figura 2, la ecuación (1) describe el análisis matemático y la gráfica resultante como área de trabajo en la figura 3.

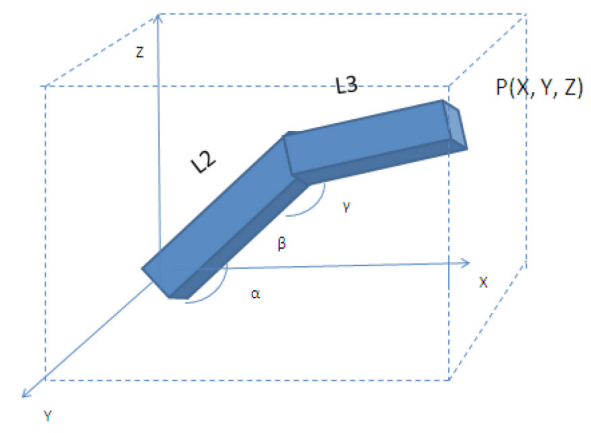

Figura 2. Análisis de cinemática directa.

$$
\begin{aligned}
& P(X)=(L 2 \cos (\beta)+L 3 \cos (\beta+\alpha)) \cdot \cos (\gamma) \\
& P(Y)=(L 2 \cos (\beta)+L 3 \cos (\beta+\alpha)) \cdot \operatorname{sen}(\gamma) \\
& P(Z)=(L 2 \cos (\beta)+L 3 \cos (\beta+\alpha))
\end{aligned}
$$

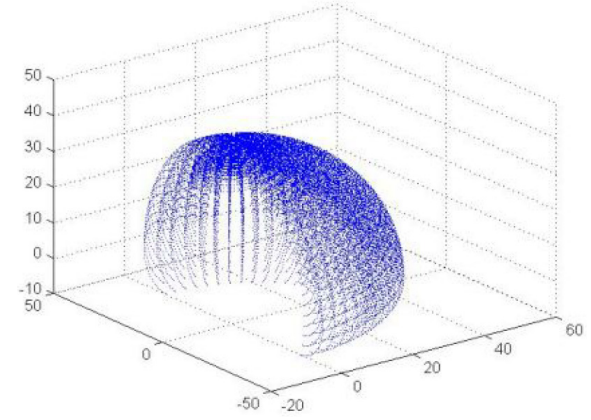

El diseño mecánico del prototipo de brazo robótico se realizó en Solid Edge versión 20 bajo licencia académica.

Para el desarrollo del diseño del prototipo se pensó en elaborar piezas sencillas, de baja complejidad y que permitieran realizar los movimientos que se requerían de acuerdo al enfoque del proyecto.

El diseño del prototipo fue pensado en una escala 1:6, la estructura está basada en el robot tipo PUMA el cual es uno de los modelos más empleados a nivel industrial, ya que es un tipo de brazo muy versátil con muy buena precisión y capacidad, pero el prototipo que se plantea es mucho más sencillo y sus piezas no son tan robustas como las de un PUMA.

Para la implementación física del prototipo del proyecto previamente se analizaron diferentes materiales teniendo en cuenta sus propiedades físicas y químicas de acuerdo a los requerimientos del proyecto en los que se determinó que los más apropiados eran el aluminio por su maleabilidad y costo y el acrílico por su rigidez y facilidad de corte láser. Las piezas que se construyeron bajo el criterio del material fueron:

Partes mecánicas en aluminio: eslabón principal del brazo que mantienen los dos servomotores principales que imprimen movimiento al hombro y antebrazo.

Partes mecánicas en acrílico: matriz de almacenamiento, caja de interfaz de usuario, soporte de cintura, soporte de antebrazo y pinza, las cuales son piezas que exigen un alto grado de precisión y exactitud con el fin de que el mecanismo funcione.

\subsubsection{Estructura mecánica en Solid Edge}

Se hizo uso de una caja como la que se observa en la figura 4 como base del robot.

Figura 3. Espacio de trabajo. 


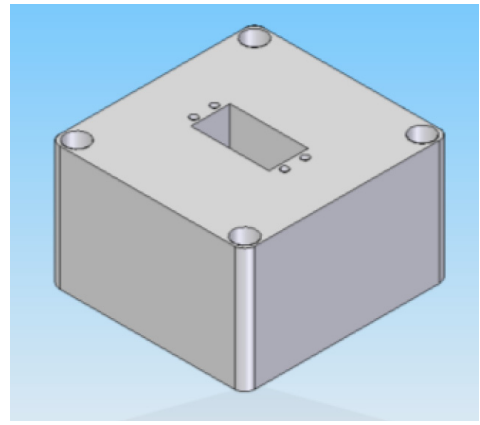

Figura 4. Base brazo robótico.

La siguiente pieza (figura 5) es el soporte de la cintura, hecha en acrílico por la rigidez del material y en forma de "U" cuadrada para estabilizar el sistema, ya que ésta es el eje principal.

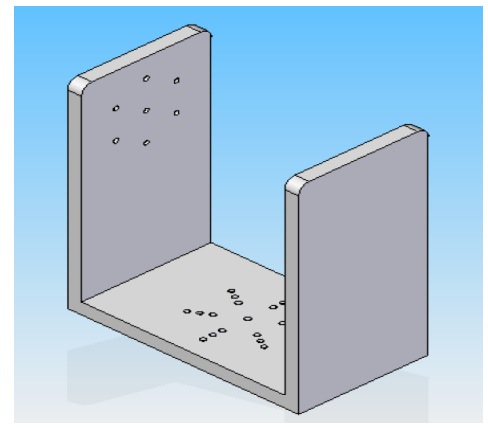

Figura 5. Cintura.

La siguiente pieza que compone el sistema es el brazo, es una pieza hecha en aluminio, adaptada para dar soporte a dos servomotores como se observa en la figura 6 que imprimen movimiento al hombro y antebrazo.

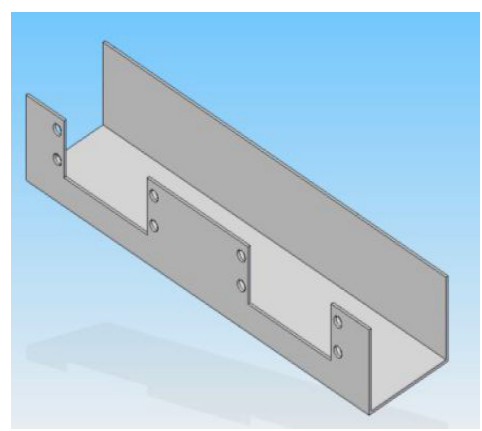

Figura 6. Brazo.

Ahora se tiene el soporte de antebrazo (figura 7), es una segunda "U" cuadrada, hecha en acrílico por la rigidez teniendo en cuenta que es una pieza que sirve como apoyo para el soporte de la pinza.

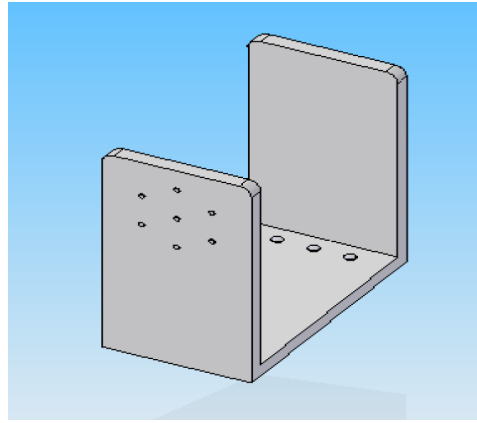

Figura 7. Antebrazo.

El soporte de la pinza (figura 8) es una pieza con un ángulo de $90^{\circ}$ y una serie de huecos que sirven como apoyo para el eje del motor y los otros para el mecanismo de los piñones en conjunto con los dedos.

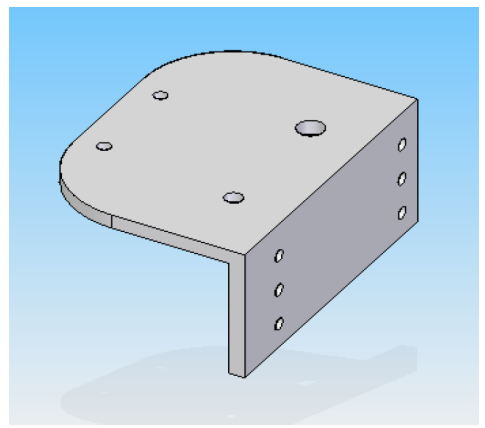

Figura 8. Base mano.

En la figura 9 se muestra un ensamble general de las piezas previamente mencionadas.

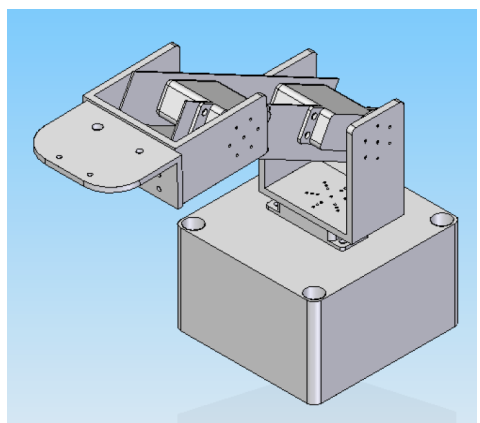

Figura 9. Ensamble general sin mano.

En la figura 10 se observa el mecanismo de la mano en el que se emplearon dos piñones, uno 
conectado al eje del servomotor y el otro haciendo eje con un tornillo sobre la plataforma del soporte y que a través de los engranajes imprimen movimiento el uno sobre el otro.

Para dar mayor estabilidad al sistema de agarre de la pinza al finalizar cada dedo se hizo uso de apoyos.

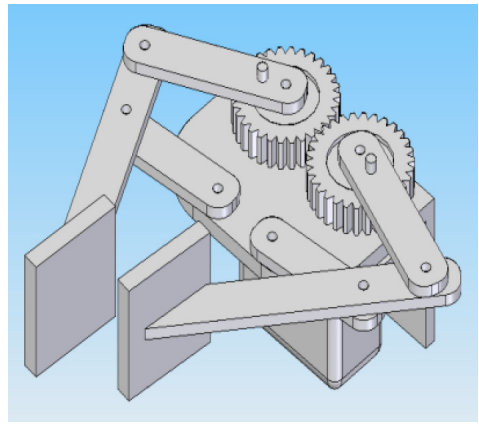

Figura 10. Mano.

El diseño final del prototipo ensamblado se observa en la figura 11.

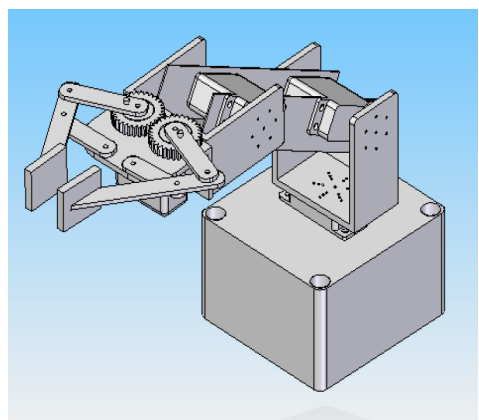

Figura 11. Estructura final.

\subsection{Desarrollo electrónico}

El programa desarrollado para el funcionamiento del brazo, se hizo teniendo en cuenta que debe posicionar cajas en una matriz y debía ofrecer características como:

- Entorno amigable, razón por la cual se empleó una LCD de $16 \times 2$ para presentar gráficamente las operaciones que esta o puede desarrollar y un teclado para que el usuario interactúe con el mismo.

- Un menú sencillo, el cual ofrece tres modos de operación como: manual, automático y protocolo de prueba.

En el diagrama de flujo de la figura 12 se presenta el diagrama general del programa.

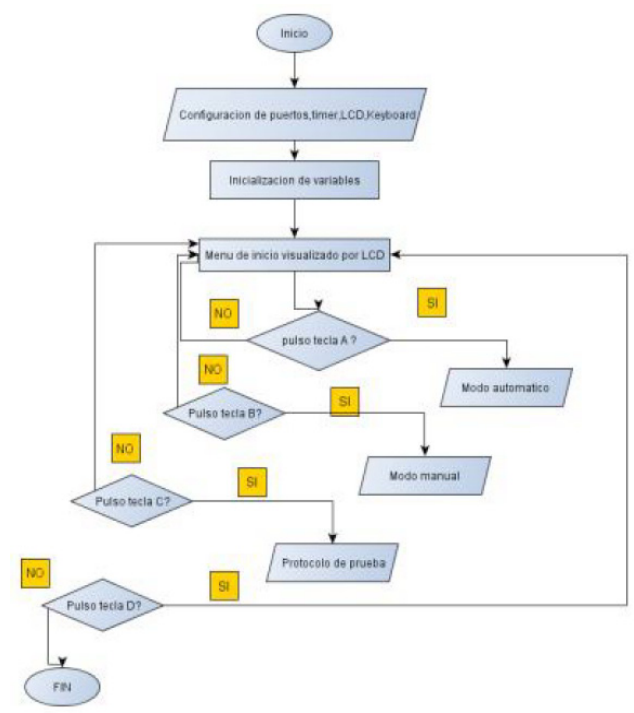

Figura 12. Diagrama general del programa.

Como se muestra en la figura 12 el programa cuenta con tres modos de operación: manual, automático y protocolo de prueba.

\subsubsection{Modo manual}

En la figura 13 se observa el diagrama de flujo, el usuario puede indicarle al brazo a través de pulsadores el posicionamiento de cada uno de los servos para que el robot adopte la posición.

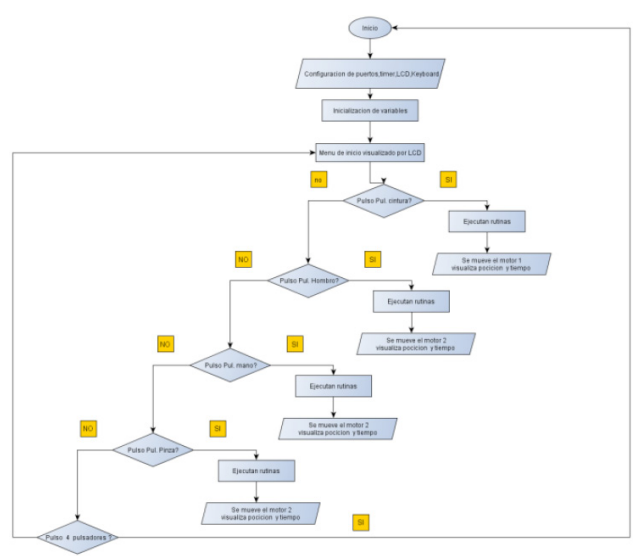

Figura 13. Diagrama de flujo modo manual. 
El circuito electrónico para el modo manual se observa en la figura 14 y en la tabla 1 se describen los componente.

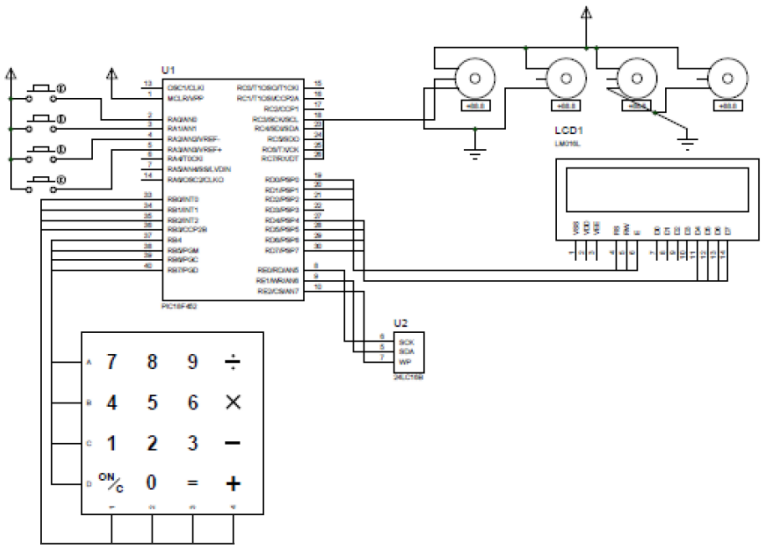

Figura 14. Circuito modo manual.

Tabla 1. Componentes del circuito manual.

\begin{tabular}{|c|c|c|c|}
\hline Materiales & Cant. & ID & Referencia \\
\hline Condensadores & 2 & $\mathrm{C} 1, \mathrm{C} 2$ & $22 \mathrm{pf}$ \\
\hline Potenciómetro & 1 & PV1 & $1 \mathrm{~K} \Omega$ \\
\hline \multicolumn{4}{|c|}{ Circuitos integrados } \\
\hline Microcontrolador & 1 & U1 & PIC18F452 \\
\hline Memoria I2C & 1 & $\mathrm{U} 2$ & 24LC16B \\
\hline Optocoplador & 4 & U3, U4, U5, U6 & MCT6 \\
\hline \multicolumn{4}{|c|}{ Motores } \\
\hline Servomotor & 4 & & $\begin{array}{c}\text { Power HD } \\
1505\end{array}$ \\
\hline \multicolumn{4}{|c|}{ Conectores y swiches } \\
\hline Pulsadores & 4 & P1, P2, P3, P4 & \\
\hline Swich on y off & 1 & & \\
\hline \multicolumn{4}{|c|}{ Otros } \\
\hline Teclado matricial & 1 & & \\
\hline Pantalla LCD & 1 & LCD1 & LM016L \\
\hline Cristal & 1 & $\mathrm{X} 1$ & $4 \mathrm{MHz}$ \\
\hline Adaptador AC-DC & 1 & & $5 \mathrm{~V}$ \\
\hline Baterías & 4 & & $1,5 \mathrm{~V}$ \\
\hline
\end{tabular}

\subsubsection{Modo automático}

En éste modo de operación las acciones ya es- tán predefinidas, éste modo tiene la función de almacenar las cajas en una matriz como la que se muestra en la figura 15 .

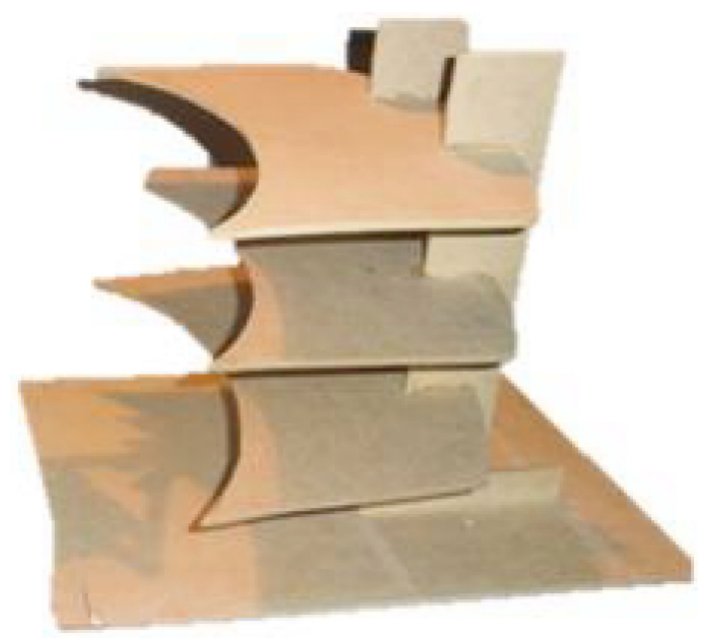

Figura 15. Matriz de almacenamiento.

En la figura 16 se observa el diagrama de flujo para el modo de operación automático.

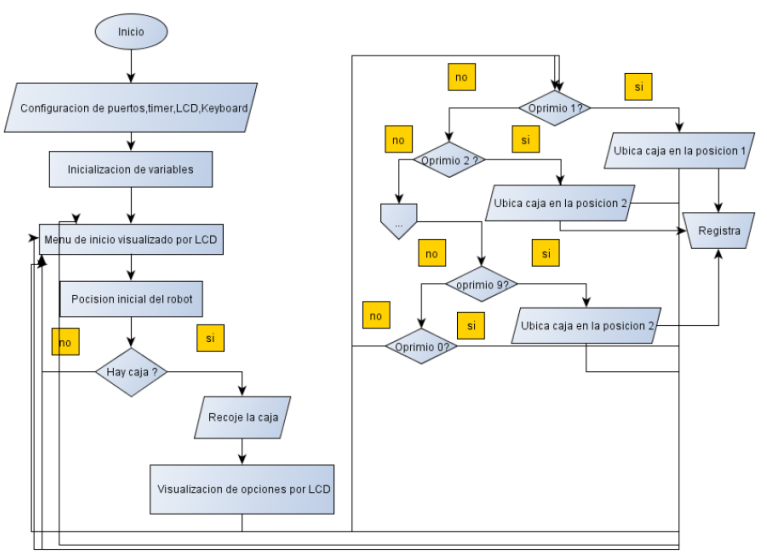

Figura 16. Diagrama de flujo modo automático.

El programa es simple como se observa en la figura 16, la caja debe estar en el área de carga, el usuario debe seleccionar el modo de operación del robot para generar el pulso con el fin de que el robot recoja la caja, para que el robot haga el respectivo procedimiento para las nueve posiciones se capturan las entradas del teclado para generar el PWM, el circuito se observa en la figura $17 \mathrm{y}$ los componentes en la tabla 2. 


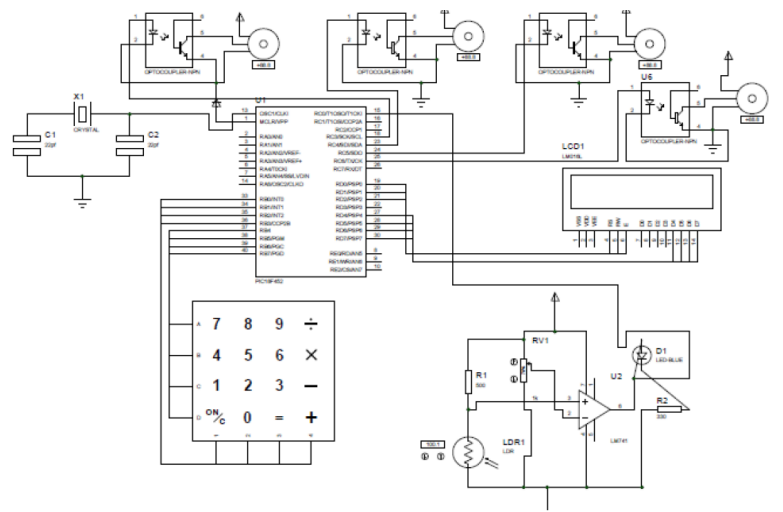

Figura 17. Circuito de control automático.

Tabla 2. Componentes del modo automático.

\begin{tabular}{|c|c|c|c|}
\hline Materiales & Cant. & ID & Referencia \\
\hline Condensadores & 2 & $\mathrm{C} 1, \mathrm{C} 2$ & $22 \mathrm{pf}$ \\
\hline \multicolumn{4}{|c|}{ Resistencias } \\
\hline \multirow{2}{*}{ Resistencias } & 1 & $\mathrm{R} 1$ & $500 \Omega$ \\
\hline & 1 & $\mathrm{R} 2$ & $330 \Omega$ \\
\hline Potenciómetro & 1 & PV1 & $1 \mathrm{~K} \Omega$ \\
\hline \multicolumn{4}{|c|}{ Diodos } \\
\hline LED & 1 & D1 & \\
\hline \multicolumn{4}{|c|}{ Circuitos integrados } \\
\hline Microcontrolador & 1 & $\mathrm{U} 1$ & PIC18F452 \\
\hline Amp. Operacional & 1 & $\mathrm{U} 2$ & LM741 \\
\hline Optocoplador & 4 & $\mathrm{U} 3, \mathrm{U} 4, \mathrm{U} 5, \mathrm{U} 6$ & MCT6 \\
\hline \multicolumn{4}{|c|}{ Motores } \\
\hline Servomotor & 4 & & $\begin{array}{c}\text { Power HD } \\
1505\end{array}$ \\
\hline \multicolumn{4}{|c|}{ Conectores y swiches } \\
\hline Swich on y off & 1 & & \\
\hline \multicolumn{4}{|c|}{ Otros } \\
\hline Teclado matricial & 1 & & \\
\hline Pantalla LCD & 1 & LCD1 & LM016L \\
\hline Cristal & 1 & $\mathrm{X} 1$ & $4 \mathrm{MHz}$ \\
\hline Fotoresistencia & 1 & LDR1 & \\
\hline
\end{tabular}

\section{RESULTADOS}

El tiempo promedio de ejecución es de 2,42 minutos, en la tabla 3 se muestra la efectividad del prototipo y en la tabla 4 los resultados de la prueba de capacidad.
Tabla 3. Efectividad de posicionamiento

\begin{tabular}{|l|c|c|c|}
\hline \multicolumn{1}{|c|}{ MOTIVO } & BAJO & MEDIO & ALTO \\
\hline $\begin{array}{l}\text { Llega y ubica la caja } \\
\text { entre el rango indicado }\end{array}$ & $85,71 \%$ & $90,47 \%$ & $100 \%$ \\
\hline $\begin{array}{l}\text { Choca pero ubica la } \\
\text { caja }\end{array}$ & $14,28 \%$ & $4,76 \%$ & $0 \%$ \\
\hline $\begin{array}{l}\text { Choca y no llega a la } \\
\text { plataforma }\end{array}$ & $4,76 \%$ & $4,76 \%$ & $0 \%$ \\
\hline
\end{tabular}

Tabla 4. Capacidad.

\begin{tabular}{|c|c|c|c|}
\hline PESO & BAJO & MEDIO & ALTO \\
\hline $10 \mathrm{~g}$ & $100 \%$ & $100 \%$ & $100 \%$ \\
\hline $20 \mathrm{~g}$ & $100 \%$ & $100 \%$ & $100 \%$ \\
\hline $30 \mathrm{~g}$ & $100 \%$ & $100 \%$ & $100 \%$ \\
\hline $40 \mathrm{~g}$ & $85,71 \%$ & $85,71 \%$ & $85,71 \%$ \\
\hline $50 \mathrm{~g}$ & $100 \%$ & $100 \%$ & $71,42 \%$ \\
\hline $60 \mathrm{~g}$ & $100 \%$ & $100 \%$ & $0 \%$ \\
\hline $70 \mathrm{~g}$ & $100 \%$ & $71,42 \%$ & $0 \%$ \\
\hline $80 \mathrm{~g}$ & $100 \%$ & $28,57 \%$ & $0 \%$ \\
\hline TOTAL & $98 \%$ & $86 \%$ & $57 \%$ \\
\hline
\end{tabular}

\section{DISCUSIÓN}

Durante el desarrollo del proyecto se observó que pese a que los motores seleccionados cuentan con un muy buen torque hay dos motores que sufren severamente como lo son el motor que se encarga de imprimir movimiento a la cintura y el motor que se encarga de mover el hombro del brazo, éste último es el más afectado ya que tiene a su cargo el peso de toda la estructura del brazo; durante el desarrollo del prototipo se identificó que fue uno de los motores que mayor problema presentó para estabilizarse ya que el ruido que el micro devolvía sobre ésta hacia que vibrara continuamente, pero gracias al uso de opto acopladores y garantizándole una buena alimentación en corriente se pudo atenuar el impacto del ruido sobre la señal de alimentación.

Otro de los grande problemas que se encontraron en el desarrollo tanto mecánico como el electrónico fue en el diseño de la pinza, porque a nivel mecánico tuvo que buscarse un modelo fácil de implementar y que cumpliera la función 
de agarrar con precisión, para el mecanismo se implementaron dos piñones uno sobre el eje del motor y en conjunto con el otro piñón imprimir movimiento al mecanismo para que con los apoyos de los otros ejes sobre la el soporte de la pinza realice la función de agarre.

Durante el desarrollo del proyecto se observó que el mecanismo de la mano se desengrana luego de realizar siete veces la opción de abrir y cerrar la mano, el mecanismo funciona luego de realizar los respectivos ajuntes sobre el engranaje de los piñones. Otro de los aspectos importantes a tener en cuenta es que el mecanismo de los dedos se desestabiliza cuando los engranajes se corren.

Cuando el robot se usa aproximadamente treinta veces en operación, las arandelas de soporte entre pieza y servo se ruedan imposibilitando al brazo realizar la función programada, para ello se recomiendan tener armillas de repuesto.

El motor que se encarga de dar movimiento al antebrazo para posicionar la pinza presenta inestabilidad en las posiciones medias, el motor se estabiliza pero tarda unos minutos en hacer y en ocasiones por la inestabilidad suele dejar de agarrar la caja.

\section{CONCLUSIONES}

Para el diseño de las piezas se tuvieron que realizar varios análisis teniendo en cuenta las fuerzas que ejerce el robot en conjunto con el área electrónica con el fin de determinar la escala a trabajar el diseño, y seleccionar el tipo de material a emplear para las piezas.

Teniendo en cuenta el momento de fuerza que imprime un motor sobre el eje que proporciona la potencia al mecanismo, se consideró que la mejor forma de distribuir y manejar el torque para el brazo era realizando una pieza central que se denominó brazo para que en ella estuvieran los dos servos encargados de imprimir movimiento al hombro y al antebrazo.

El presente trabajo generó problemas cinemáticos y electrónicos para el desarrollo del manipulador robótico, por lo tanto al diseñar la etapa de control del brazo se tuvo en cuanta cierto tipo de posiciones para realizar diferentes cantidades de operaciones en el modo manual , independiente del modo automático esto se debe a que son operaciones únicas necesarias para el cumplimento de los objetivos planteados , no solo la cantidad de operaciones son constantes , sino que se trata de una cifra mucho menor a la planteada a las posibilidades que da el modo manual.

En el diseño electrónico se obtuvieron los objetivos propuestos; cada modo de utilización del brazo robótico fue lo suficiente mente adecuado para poder tomar las piezas o cajas y no soltarlas hasta el lugar asignado. Así también la tarjeta electrónica diseñada, cumplió con las expectativas de actuar como interfaz entre el usuario y los servomotores del manipulador a su vez que integraba todo el sistema electrónico.

El comportamiento de los servomotores no fue siempre constante después de intentos se llegó que el ruido del microcontrolador al enviar los pulsos interfería y hacia que la funciones que deseábamos realizar variaran para esto fue importante crear una etapa de aislamiento por medio de opto acopladores y cable blindado par la conexión de los motores.

El desempeño general del Brazo Manipulador, que es el objetivo de este trabajo, fue en general muy bueno, logrando cumplir con todas las metas propuestas desde su concepción. 


\section{Referencias Bibliográficas}

[1] Diario el Tiempo; Los Discapacitados son una prioridad" Diario el Tiempo. [En línea], consultado en Mayo 15 de 2011, disponible en: http://www.eltiempo.com/ archivo/documento/MAM-2198901.

[2] J. Snehi; Computer peripherals and interfacing, Editorial: Laxmi publications LTDA, Primera edición, 2006.
[3] A. Ollero; Robótica: manipuladores y robots móviles", Editorial Marcombo, 2001.

[4] J. Craig; Robótica, Editorial: Pearson educación, 2006.

[5] A. Serway, Física, Editorial: Pearson Educación, 2001. 